\title{
Эволюция управления бизнес-процессами и реализация инициатив по совершенствованию бизнес-процессов
}

\author{
Р. Г. Пожидаев ${ }^{1 凶}$ \\ ${ }^{1}$ Воронежский государственный университет, Университетская пл, 1, \\ 394018, Воронеж, Российская Федерация
}

Для цитирования: Пожидаев Р. Г. Эволюция управления бизнес-процессами и реализация инициатив по совершенствованию бизнес-процессов // Вестник Воронежского государственного университета. Серия: Экономика и управление. 2020. № 3. С. 122-132. DOI: 10.17308/econ.2020.3/3111

\begin{abstract}
Предмет. Рассматривается эволюция управления бизнес-процессами - от проектирования отдельных бизнес-процессов до самостоятельной дисциплины менеджмента и современного понимания управления бизнес-процессами как комплексного набора организационных способностей. Акцент сделан на анализе лучших практик реализации проектов по совершенствованию бизнес-процессов. Цель. Анализ современных теоретических и прикладных аспектов управления бизнес-процессами, причин провала и успеха проектов по совершенствованию бизнес-процессов, определение оптимальной организации, структуры и порядка работы проектных команд по совершенствованию бизнес-процессов.

Метод. Обзор литературы, анализ прикладных исследований и кейсов.

Результат. Определены условия организации проектных работ по совершенствованию бизнес-процессов, необходимая квалификация членов команды проекта, общая структура участников проекта по совершенствованию бизнес-процессов (команда проекта, команда поддержки (пилотная группа), комитет по проверке контрольных точек, команды подпроектов). Уточнено содержание этапов работ по совершенствованию бизнес-процессов.

Вывод. Потенциал управления бизнес-процессами и инициатив по совершенствованию бизнес-процессов может быть реализован при условии их встраивания в важнейшие компоненты управления организации, такие как стратегия, структура, организационная культура, информационно-коммуникационные технологии, управление персоналом. Не менее важно постоянное развитие компетенций: не только в анализе и моделировании бизнес-процессов, но и управлении проектами и организационными изменениями.
\end{abstract}

Ключевые слова: управление бизнес-процессами, совершенствование бизнес-процессов, команда проекта.

\section{Введение}

В последние десятилетия благодаря широкому практическому применению концепции и методы совершенствования бизнес-процессов а также программное обеспечение для описания и моделирования бизнес-процессов, постоянно развивались и оттачивались. Однако, несмотря на обширный и доступный инструментарий управления бизнес-процессами, нельзя сказать, что проекты по совершенствованию бизнес-процессов в общем случае реа- лизуются успешно, с достижением поставленных целей и планируемых эффектов. Основные сложности связаны с управлением бизнес-процессами как стратегически важной системой организации, грамотной организацией работы команды проекта, своевременным изменением ее состава в зависимости от стадии проекта, обеспечением проектов необходимыми ресурсами, бюджетом и поддержкой. За последнее время проведено достаточно аналитических исследований причин провала и успеха проек-

(c) Пожидаев Р. Г., 2020

Вестник ВГУ. Серия: Экономика и управление. 2020. № 3. С. 122-132. 
Эволюция управления бизнес-процессами и реализация инициатив...

тов по совершенствованию бизнес-процессов, что позволяет определить оптимальную организацию и порядок работы проектных команд. Основная цель данной статьи - представить данный опыт, а также рассмотреть современные теоретические и прикладные аспекты управления бизнес-процессами.

Несмотря на то что основные концепции реинжиниринга и совершенствования бизнес-процессов ведут свою историю с конца 1980-х гг., управление бизнес-процессами (Business Process Management, BPM) долгое время воспринималось как расширение управления потоками работ (Workflow Management, WFM), а затем как часть ERP-систем (Enterprise resource planning), таких как SAР и Oracle (позже и как часть программного обеспечения для интеграции и инфраструктуры приложений, например, IBM WebSphere и Cordys Business Operations Platform) $[5 ; 10 ; 17]$.

Однако практика показала, что совершенствование бизнес-процессов играет важную роль в управлении бизнесом и не ограничивается управлением данными и операциями. С начала 2000-х гг. управление бизнес-процессами постепенно превращается в самостоятельную дисциплину, которая объединяет знание информационных технологий и знание науки управления и применяет их к операционным бизнес-процессам. Управление бизнес-процессами стало рассматриваться как организационная система, обеспечивающая непрерывный жизненный цикл и постоянное совершенствование бизнес-процессов, задачи которой - оптимизация ресурсов, повышение эффективности деятельности при снижении затрат, сокращение времени выполнения заказов и повышение рентабельности инвестиций [7; 8; 13].

Сегодня управление бизнес-процессами развилось в комплексную дисциплину менеджмента с акцентом на организационные процессы и воспринимается как сложная совокупность корпоративных способностей, непосредственно связанных со стратегией, системой управления, информационно-коммуникационными технологиями, персоналом и организационной культурой $[1 ; 6 ; 18]$.

Управление бизнес-процессами должно способствовать достижению стратегических целей организации. Соответствующие способности включают оценку процессов и инициатив по совершенствованию бизнес-процессов на их соответствие общей корпоративной стратегии. Управление бизнес-процессами сегодня все больше интегрируется в систему корпора- тивного управления и отчетности, прежде всего через определение и измерение ключевых показателей эффективности деятельности.

Несмотря на хорошо разработанные методы, стандарты и инструменты, организация работы по совершенствованию бизнес-процессов является одной из самых сложных как с точки зрения организационных изменений, так и с точки зрения управления проектами.

\section{Метод}

\section{Организационные основы управления бизнес-процессами}

В отечественной литературе в качестве организационной основы управления бизнес-процессами часто указывают модели создания ценности (или, как их часто называют, эталонные процессные модели $)^{1}$, не заслуженно обходя вниманием концепцию конкурентных приоритетов.

В конце 1980-х гг. ряд авторов сформулировали концепцию конкурентных приоритетов, которая впоследствии стала предпосылкой определения состава бизнес-процессов, направлений их совершенствования и измерения эффективности бизнес-процессов [3; 9]. Концепция конкурентных приоритетов исходит из того, что чаще всего успех в конкурентной борьбе основан на приобретении преимуществ по времени, качеству, стоимости и гибкости.

Время в данном случае - показатель длительности всего производственного цикла или отдельного бизнес-процесса. Сокращение времени подразумевает сведение к минимуму таких показателей, как время в очереди и время ожидания. Время в очереди определяется как время, когда производственный процесс или бизнес-процесс простаивает по причине отсутствия необходимых или свободных ресурсов для его продолжения. Время ожидания определяется как время, когда процесс проста-

${ }^{1} \mathrm{~K}$ числу наиболее распространенных моделей создания ценности (эталонных процессных моделей) относят: цепочку создания ценности М. Портера; модель IBL (The International Business Language); 13-процессную модель, разработанную Американским центром производительности и качества (American Productivity and Quality Center); 8-процессная модель; модель BAAN; модель ITIL/ITSM; и др.

Эталонные модели процессов создаются в результате обобщения соответствующего мирового опыта и существуют главным образом в форме международных стандартов, разрабатываемых международной организацией по стандартизации (ISO) и другими авторитетными международными и национальными организациями. 
ивает не только несмотря на наличие необходимых ресурсов, но по причине отсутствия синхронизации или последовательности с другими процессами.

Исходя из определения качества как степени, в которой набор неотъемлемых характеристик удовлетворяет требованиям, в данном контексте присущие характеристики определяют качество процесса или системы, связанных с определенными требованиями. Поэтому качество измеряется по отношению к требованию и не является абсолютным показателем.

Cтоимость (расходы) в данном контексте не имеет выраженной специфики и кроме стандартного деления (на прямые и косвенные затраты, фиксированные и переменные расходы и т. п.), скорее, является взаимозависимой с другими измерениями (например, стоимость качества как расходы на сбои (отказы), оценку, профилактику).

Гибкость, как способность реагировать на изменения, в рассматриваемом контексте является способом поддержания соответствия между бизнес-процессами и системой управления организацией в меняющихся условиях (что можно назвать внешней гибкостью), а также возможностью оперативно изменять бизнес-процесс в целях повышения его результативности (внутренняя гибкость).

Таким образом, управление бизнес-процессами объединяет техническую (информационную) составляющую - моделирование и автоматизацию бизнес-процессов, и организационную составляющую, основанную на эталонных моделях процесса и концепции конкурентных приоритетов.

Исходя из отрасли, специфики деятельности и задач организационного развития управление бизнес-процессами, следует определить методы, технологии и инструменты совершенствования бизнес-процессов и адаптировать их к конкретным требованиям организации. В современной практике бизнеса управление бизнес-процессами, как правило, реализуется посредством процессно-ориентированных информационных систем ${ }^{2}$, которые объединяют опе-

${ }^{2}$ Термин «процессно-ориентированная информационная система» (process-aware information systems, PAIS) объединяет различные типы базовых корпоративных информационных систем, таких как системы управления потоками работ (Workflow Management, WFM), системы планирования ресурсов предприятия (Enterprise resource planning, ERP), корпоративные системы управления контентом (Enterprise content management, ECM), системы мониторинга деловой активности (Business activity monitoring, ВАМ) и др. рации и технологии, персонал и функции, источники информации и информационные потоки. Инновации в проектировании и функционировании процессно-ориентированных информационных систем позволяют реорганизовать бизнес-процессы с высокой степенью адаптивности за счет реализации технологий объектно-ориентированного проектирования, систем групповой работы, систем поддержки принятия решений и систем управления знаниями. Однако необходимо помнить, что любая система не определяет появление и поведение конкурентов, изменение предпочтений и лояльность клиентов, стратегические цели и задачи, поэтому выбор целесообразных методов, систем и инструментов является важнейшей задачей как общего руководства организации, так и управления бизнес-процессами. Ошибки могут стоить компании больших финансовых затрат, зря потраченных усилий и времени.

Приступая к реорганизации бизнес-процессов, необходимо сопоставить возможности совершенствования со способностями организации реализовать подобные инициативы - прежде всего с точки зрения их обеспечения финансовыми ресурсами и наличием персонала, обладающего соответствующими компетенциями. Задача руководства компании - на основе статистики (например, совокупности негативных отзывов клиентов) и оценки ресурсов определить небольшое и достижимое число бизнес-процессов, самым непосредственным образом влияющих на достижение целей и задач организации [16; 20].

\section{Организация работ по совериенствованию бизнес-процессов}

При формировании команды проекта необходимо, чтобы ее члены имели квалификацию и опыт:

- в методах моделирования, проектирования и анализа бизнес-процессов;

- методах проведения исследований, групповой работе, интервьюировании;

- методах выстраивания коммуникаций и управления организационными изменениями;

- понимании задач функциональных подразделений, а также деятельности всех подразделений, которые затрагивает совершенствование бизнес-процесса;

- обучении и инструктировании персонала.

Необходимо определить, кто из членов команды будет работать изо дня в день только над данным проектом, а кто будет совмещать участие в проекте с работой в своем подразделении. 
Эволюция управления бизнес-процессами и реализация инициатив...

Чаще всего организационная структура проекта включает только команду проекта, однако практика бизнеса показала полезность команды поддержки (или пилотной группы), т. е. группы, которая будет апробировать изменения, перед тем как внедрить бизнес-процесс. Эта группа должна быть отделена от команды проекта, хотя и работать с ней в постоянном контакте. Она играет важную роль в качественном тестировании, выявлении рисков, построении системы коммуникаций и информационных потоков процесса. Соответственно, участники команды поддержки должны быть мотивированы к активному участию в совершенствовании бизнес-процесca $[11 ; 12 ; 15]$.

Некоторые специалисты считают целесообразным создание команды (комитета) по проверке контрольных точек - фактически группы экспертов, занимающихся проверкой предлагаемых решений, выявлением рисков и дающих рекомендации по реализации проекта [14; 16]. Данная группа также должна действовать отдельно от команды проекта. Стоит отметить, что не все компании располагают достаточным числом специалистов по управлению бизнес-процессами, чтобы формировать и группу экспертов, и команду проекта.

Еще одна задача, которой часто не уделяется достаточного внимания, - это управление ожиданиями персонала относительно изменений. Изменения часто воспринимаются негативно, безотносительно их реальной пользы и эффективности. Посредством регулярного и честного общения необходимо создать атмосферу уверенности работников в том, что изменения не направлены против них, правильно организовать обучение и инструктирование, дать им возможность принять изменения, которые повлияют на их деятельность.

Собственно работу по совершенствованию бизнес-процесса оптимально начинать со встречи команды проекта, на которой будет также присутствовать руководство компании, владелец процесса, руководители или представители ключевых заинтересованных сторон (внутри организации, а возможно, и вне её - например, представители ключевых поставщиков или заказчиков). Стартовая встреча позволяет:

- сообщить о важности инициативы и ее влиянии на организацию;

- представить и обсудить цели проекта и ожидаемые преимущества его реализации для организации;
- познакомиться членам команды друг с другом, тем самым повышая уровень их комфорта и уверенность в командной работе;

- ответить на вопросы и решить организационные проблемы;

- установить сроки и формат отчетов о состоянии работ и процедуры регулярных коммуникаций;

- сформулировать процедуры принятия решений в команде проекта.

Проекты по совершенствованию бизнес-процессов обычно состоят из шести этапов $[4 ; 14 ; 19]^{3}$ :

- понимание проекта;

- анализ бизнес-процесса;

- перепроектирование (совершенствование) бизнес-процесса;

- внедрение усовершенствованного бизнес-процесса;

- развертывание (выполнение) усовершенствованного бизнес-процесса;

- оценка и измерение бизнес-процесса.

1. Понимание задач и содержания проекта.

Первый этап включает следующие основные виды работ:

- разработка описания проблем, которые должны быть решены;

- разработка предварительного плана проекта, в том числе ключевых контрольных точек проекта;

- определение всех заинтересованных сторон процесса;

- определение полного состава команды проекта;

- определение предварительного объема работ по проекту;

- определение состава желаемых улучшений в процессе;

- определение потенциальных рисков проекта;

- подготовка предварительного экономического обоснования для изменений в процессе;

\footnotetext{
${ }^{3}$ К числу наиболее распространенных моделей создания ценности (эталонных процессных моделей) относят: цепочку создания ценности М. Портера; модель IBL (The International Business Language); 13-процессную модель, разработанную Американским центром производительности и качества (American Productivity and Quality Center); 8-процессная модель; модель BAAN; модель ITIL/ITSM; и др.

Эталонные модели процессов создаются в результате обобщения соответствующего мирового опыта и существуют главным образом в форме международных стандартов, разрабатываемых международной организацией по стандартизации (ISO) и другими авторитетными международными и национальными организациями.
} 
- разработка концепции видения процесса «как должно быть»;

- разработка предварительного плана коммуникаций (членов команды с заинтересованными сторонами);

- установка предварительных сроков и бюджетов;

- определение потребностей в обучении;

- разработка предварительного плана внедрения усовершенствованного бизнес-процесса.

На первом этапе важно определить все заинтересованные стороны, т. е. всех, кто будет затронут реорганизуемым процессом. Заинтересованные стороны могут быть прямыми и косвенными. Косвенные заинтересованные стороны - это сотрудники и подразделения (часто выполняющие административную работу), принимающие участие в сборе или формализации информации, используемой в ходе выполнения бизнес-процесса, являющиеся получателями информации о результатах процесса, а также не включенные в выполнение бизнес-процесса, но контактирующие с клиентами компании по вопросам, связанным с выполнением или результатами бизнес-процесса $[2 ; 18]$.

С точки зрения состава команды проекта главную роль на первом этапе будут играть ее члены с опытом анализа и проектирования бизнес-процессов, а также представители ключевых подразделений и бизнес-единиц, непосредственно участвующих в выполнении бизнес-процесса, способные предоставить максимум информации о проблемах и желаемых направлениях совершенствования бизнес-процесса.

2. Анализ бизнес-процесса «как есть». Второй этап фокусируется на внимательном изучении процесса (включая подпроцессы), который должен быть изменен. Необходимо собрать информацию о текущем («как есть») процессе различными способами, в том числе с помощью:

- интервью;

- фокус-групп;

- опросов;

- наблюдения.

Модель процесса «как есть» позволит определить причины проблем и сбоев, возможности совершенствования бизнес-процесса.

Кроме специалиста по анализу и проектированию бизнес-процессов, на этом этапе ведущими членами команды проекта будут разработчик моделей процессов, организатор и модератор фокус-групп. Желательно участие на данном этапе экспертов и консультантов (комитета по проверке контрольных точек) как из числа сотрудников компании, так и (при возможности) приглашенных извне.

Работа команды проекта с персоналом компании на данном этапе часто ограничивается опросами и наблюдением, но интервью и фокус-группы равно важны для выявления идей и предложений сотрудников, непосредственно выполняющих бизнес-процесс.

После описания процесса «как есть» необходимо тщательно проверить его с теми, кто выполняет бизнес-процесс, чтобы убедиться, что все операции, информационные и материальные потоки включены и расставлены в правильной последовательности.

3. Проектирование бизнес-процесса «как должно быть». Это этап сбора и анализа идей и их воплощения в варианты совершенствования бизнес-процесса. На данном этапе важно создать и использовать максимально широкую информационную базу - как идей и предложений всех заинтересованных сторон, так и внешней информации, прежде всего:

a) каковы лучшие из существующих практик выполнения подобных процессов?

б) какие инновационные идеи и практики используют конкуренты?

Команда проекта работает над следующими основными задачами:

- определение критериев для нового процесса и расстановка приоритетов (относительной важности критериев) на основе потребностей компании;

- разработка вариантов процесса «как должно быть» и их представление заинтересованным сторонам.

В рамках данного этапа возможные варианты процесса «как должно быть» сужаются до 2-3 путем обсуждений с заинтересованными сторонами и проведения мозговых штурмов. Оставшиеся варианты проходят верификацию на основе ответов на следующие вопросы:

а) какой процесс, если он будет реализован, будет отвечать всем потребностям заинтересованных сторон и целям компании?

б) какой процесс наилучшим образом соответствует технологии, оборудованию и уровню квалификации его исполнителей?

в) кроме того, необходимо учитывать потребности в обучении, бюджет на внедрение и потенциальные риски внедрения.

Важнейшую роль играют заинтересованные стороны, и задача команды проекта - обеспечить результативное обсуждение с заинтере- 
сованными сторонами вариантов совершенствования бизнес-процесса.

Кроме мозговых штурмов и обсуждений 2-3 вариантов бизнес-процесса, оптимально, уже на данном этапе, сформировать и задействовать команду поддержки для их апробации.

Выбор варианта усовершенствованного бизнес-процесса происходит после утверждения заинтересованными сторонами и руководства компании.

Третий этап завершается составлением обновленного плана проекта, включающего;

- уточненный план внедрения бизнес-процесса;

- план обучения и инструктирования персонала;

- план реализации подпроектов;

- бюджет внедрения бизнес-процесса и обучения сотрудников;

- потенциальные риски внедрения.

4. Внедрение усовершенствованного процесса. Четвертый этап подразумевает изменения в команде проекта, появление подпроектов и активную работу команды поддержки.

Изменения в команде проекта связаны с выдвижением на ведущие позиции тех, кто будет контролировать реализацию процесса. Важно подобрать команду реализации как из представителей функциональных подразделений и бизнес-единиц, так и членов команды проекта, понимающих процесс в целом - для достижения баланса общего видения и необходимой детализации работ.

Выполнение подпроектов целесообразно на данной стадии. Как правило, подпроекты связаны:

- с изменениями в программном обеспечении и информационной системе компании;

- выделением кадровых ресурсов для разработки новых ролей и обязанностей, а также для обучения новому процессу;

- разработкой положений и процедур, связанных с новым процессом.

Команды подпроектов, как правило, создаются из представителей ответственных подразделений и членов команды основного проекта. Для руководителя проекта это весьма сложный этап - необходимо обеспечить обмен информацией и координацию между командой проекта и командами подпроекта, осуществлять постоянный контроль и решать проблемы, соблюдать требуемые сроки и бюджеты, оценивать полученные результаты. Важно добиться единых процедур и форматов в отношении тестирования, отчетов о состоя- нии и прочей документации. Кроме того - не забывать о доступности и постоянном обмене информацией команд подпроектов с заинтересованными сторонами.

Команда поддержки (пилотная группа) должна пройти весь процесс (включая подпроцессы) от начала до конца в поисках любых проблем, которые могут возникнуть. Не стоит недооценивать значение правильного и тщательного тестирования нового процесса. Не стоит также бояться затрат времени (как правило больших) на работу команды поддержки необходимо сделать все для качественного развертывания и внедрения процесса в масштабах всей компании. Вполне возможно, что придется осуществить несколько циклов проверки командой поддержки.

Данный этап завершается разработкой плана развертывания процесса, включающего:

- коммуникационные стратегии и методы;

- план обучения;

- график развертывания процесса;

- план оценки и измерения воздействия на деятельность компании.

5. Развертывание (выполнение) усовершенствованного бизнес-процесса. На пятом этапе в команду проекта включаются члены команды поддержки, которые до этого действовали независимо. Они становятся проводниками изменений для своих коллег, так как они могут ответить на возникающие вопросы и решить проблемы сотрудников, осваивающих новый бизнес-процесс.

Развертывание усовершенствованного процесса усложняется тем, что реализуются как основной процесс, так и результаты подпроцессов, что требует вовлечения большой группы сотрудников организации и масштабного обучения.

Собственно обучение является основным содержанием данного этапа. Требуется убедиться, что обучение предоставляется всем, кто будет вовлечен в исполнение нового процесса, что это обучение включает все необходимые методологические и практические составляющие.

6. Оценка и измерение. Шестой этап подразумевает определение ответственных за мониторинг и оценку достижения планируемых параметров и критериев бизнес-процесса. Это могут быть как члены проектной команды, так и новая межфункциональная команда, состоящая из представителей подразделений, непосредственно выполняющих бизнес-процесс. В их задачи также входит выявление проблем, 
возникающих по мере выполнения бизнес-процесса, поиск их решений и дальнейшее совершенствование бизнес-процесса.

Так как контрольные точки бизнес-процесса были определены на предшествующих этапах проекта, в ходе мониторинга важно получить статистически обоснованные данные о точности определения и необходимом числе контрольных точек и параметров измерения бизнес-процесса.

Когда статистика результатов выполнения нового бизнес-процесса за определенный период будет собрана, можно сделать заключение об эффективности реализации проекта и дать оценку работы команды проекта. Оптимально это осуществить в рамках совещания по подведению итогов проекта с участием представителей всех заинтересованных сторон и сотрудников, участвовавших в реализации проекта на разных этапах. Кроме того, важно зафиксировать положительные и отрицательные стороны организации проекта, чтобы использовать в дальнейшем лучшие практики и извлечь уроки из ошибок.

\section{Обсуждение результатов}

Организация работы по совершенствованию бизнес-процессов является одним из самых сложных видов деятельности как с точки зрения организационных изменений, так и с точки зрения управления проектами. Это происходит по ряду причин, основные из которых следующие.

1. Разработка системы бизнес-процессов охватывает и влияет на деятельность всей организации, при этом не только для совершенствования бизнес-процессов, но и для оценки правильности и эффективности данной работы требуется значительное время.

2. Организация является развивающейся системой, действующей в меняющемся деловом окружении, т. е. деятельность организации не будет стабильной весь период работ по совершенствованию бизнес-процессов. Изменения могут существенно влиять на приоритеты работы над бизнес-процессами, также как и меняться временные рамки и набор необходимых ресурсов для реализации подобных инициатив.

3. Совершенствование бизнес-процессов является проектом, а результатом работы - процесс, который продолжает изменяться в ходе развития компании. Это создает сложности в организации и управлении подобными работами, так как руководитель и команда проекта должны понимать указанные две составляющие и определить правильный баланс проектной работы (которая должна быть завершена в определенные сроки и с определенными результатами) и возможности дальнейшего совершенствования бизнес-процесса (что возможно, во-первых, при успешной реализации проекта, а во-вторых - при правильной организации процедур мониторинга и контроля выполнения процесса). Важность фактора времени и изменений, отмеченная в пунктах 1 и 2, имеет место и в проектной работе - в определении, какие ресурсы и в какое время целесообразно использовать для достижения цели проекта.

Процессно-ориентированное проектирование непосредственно затрагивает организационную структуру, и задача управления бизнес-процессами в данном случае состоит в совмещении требований к распределению ответственности и осуществлении контроля вдоль всего бизнес-процесса с принципами и правилами проектирования организационной структуры.

Как комплексная дисциплина управление бизнес-процессами требует не только наличия и повышения квалификации и компетенций сотрудников в экспертизе, проектировании и администрировании бизнес-процессов, но и систематического согласования с управлением человеческими ресурсами. Это важно не только потому, что методологические навыки анализа и моделирования бизнес-процессов должны быть связаны с навыками управления изменениями, но прежде всего для обеспечения обучения персонала работе с новыми бизнес-процессами, включая понимание контекста, информационной инфраструктуры и поведенческих характеристик. Наконец, управление бизнес-процессами нуждается в общей системе ценностей и организационной культуре,поддерживающей совершенствование процессов и инновации [12].

\section{Заключение}

В целом можно сделать обоснованный вывод о расширении роли управления бизнес-процессами в компаниях, использующих процессный подход. Одним из практических следствий признания этого факта является современное понимание организации работ по совершенствованию бизнес-процессов.

Компании инициируют реорганизацию бизнес-процессов по ряду причин, но цели совершенствования бизнес-процессов всегда направлены:

- на улучшение обслуживания клиентов; 
- развитие способностей предвидеть, peaгировать и соответствовать изменениям рынка и делового окружения;

- максимизацию возможностей развития бизнеса;

- рост конкурентоспособности компании и способности отвечать на вызовы конкурентов;

- снижение потерь, уменьшение числа ошибок и повышение эффективности процесса.

В рамках совершенствования отдельных бизнес-процессов могут решаться следующие задачи:

- обновление системы информационно-коммуникационных технологий компании на основе понимания взаимозависимости бизнес-процессов;

- сокращение числа операций в рамках бизнес-процесса и времени на их выполнение;

- упорядочение и повышение рациональности действий сотрудников, выполняющих бизнес-процесс;

\section{Библиографический список}

1. Aarnio T. The Strengthened Business Process Matrix - A Novel Approach for Guided Continuous Improvement at Service-Oriented SMEs // Knowledge and Process Management. 2015. Vol. 22 (3). P. 180-190, doi.org/10.1002/kpm.1473

2. Bakotich D., Krnich A. Exploring the relationship between business process improvement and employees' behavior // Journal of Organizational Change Management. 2017. Vol. 30 (4). P. 1044-1062, doi. org/10.1108/jocm-06-2016-0116

3. Clark K. Dynamic Manufacturing Creating the Learning Organization // Free Press. New York. 1988. $349 \mathrm{p}$.

4. Crawford L. Developing organizational project management capability: theory and practice // Project Management Journal. 2006. Vol. 37 (3). P. 74-97, doi. org/10.1177/875697280603700308

5. Davenport T. $H$. Process Innovation - Reengineering Work Through Information Technology // Harvard Business School Press. Boston. 1993. 352 p.

6. Grover V., Kettinger W. J., Teng J. T. C. Business process change in the 21st century // Business \& Economic Review. 2000. Vol. 46 (2). P. 14-18.

7. Grover $V$. From business reengineering to business process change management: a longitudinal study of trends and practices // IEEE Transactions on Engineering Management. 1999. Vol. 46 (1). P. 36-46.

8. Harrington H. J. Business Process Improvement The Breakthrough Strategy for Total Quality, Productivity, and Competitiveness. McGraw-Hill. New York. 1991. $274 \mathrm{p}$.
- применение лучших практик на рабочих местах и в подразделениях компании.

Таким образом, несомненный потенциал управления бизнес-процессами и инициатив по совершенствованию бизнес-процессов может быть реализован при условии их встраивания в важнейшие компоненты управления организации, такие как стратегия, структура, организационная культура, информационно-коммуникационные технологии, управление персоналом. Не менее важно постоянное развитие компетенций не только в анализе и моделировании бизнес-процессов, но и управлении проектами и организационными изменениями.

\section{Конфликт интересов}

Автор декларирует отсутствие явных и потенциальных конфликтов интересов, связанных с публикацией настоящей статьи.

9. Hayes $R$. H. Restoring Our Competitive Edge: Competing Through Manufacturing. John Wiley. New York. $1984.280 \mathrm{p}$.

10. Karimi J., Somers T., Bhattacherjee A. The impact of ERP implementation on business process outcomes: a factor-based study // Journal of Management Information Systems. 2007. Vol. 24 (1). P. 101-134.

11. Jurisch M. C., W Palka., Wolf P., Krcmar H. Which capabilities matter for successful business process change? // Business Process Management Journal. 2014. Vol. 20 (1).P.47-67, doi.org/10.1108/bpmj-11-2012-0125

12. Leyer M., Stumpf-Wollersheim J., Kronsbein D. Stains on the bright side of process-oriented organizational design: an empirical investigation of advantages and disadvantages // Schmalenbach Business Review. 2017. Vol. 17 (1). P. 29-47, doi.org/10.1007/ s41464-016-0020-9

13. Rosemann $M$. The Service Portfolio of a BPM Center of Excellence // Handbook on Business Process Management. 2010. Vol. 2. P. 267-284, doi. org/10.1007/978-3-642-01982-1_13

14. Rosemann M., Brocke vom J. The Six Core Elements of Business Process Management // Handbook on Business Process Management. 2010. Vol. 1. P. 107122, doi.org/10.1007/978-3-642-00416-2_5

15. Povey B. The Development of a Best Practice Business Process Improvement Methodology // Benchmarking for Quality Management and Technology. 1998. Vol. 5(1). P. 27-44, doi.org/10.1108/14635779810206795

16. Sarker S., Sidorova A. Understanding business process change failure: an actor-network perspective // Journal of Management Information Systems. 2006. Vol. 23 (1). P. 51-86, doi.org/10.2753/mis0742-1222230102 
17. W. van der Aalst, K. van Hee. Workflow Management: Models, Methods, and Systems // MIT Press. Cambridge. 2002. 316 p.

18. Brocke vom J., Rosemann M. Business Process Management // Wiley Encyclopedia of Management, Vol. 7. Management Information Systems. 2014. P. 11-37, doi.org/10.1007/978-3-642-45100-3
19. Brocke vom J., Sinnl T. Culture in business process management: a literature review / J.vom Brocke // Business Process Management Journal. 2011. Vol. 17 (2). P. 357-377, doi.org/10.1108/14637151111122383

20. Zaini Z., Saad A. Business Process Reengineering as the Current Best Methodology for Improving the Business Process // Journal of ICT in Education. 2019. Vol. 6. P. 66-85.
Пожидаев Руслан Геннадьевич, кандидат экономических наук, доцент, доцент кафедры экономики труда и основ управления, Воронежский государственный университет, Воронеж, Российская Федерация

E-mail: ruslan_pozhidaev@mail.ru

ORCID: 0000-0002-5297-673X
Поступила в редакцию 10.07.2020

Подписана в печать 10.09.2020 


\title{
The evolution of business process management and the implementation of business process improvement initiatives
}

\author{
Ruslan G. Pozhidaev ${ }^{1 凶}$ \\ ${ }^{1}$ Voronezh State University, Universitetskaya pl., 1, 394018, Voronezh, Russian Federation
}

Cite as: Pozhidaev, R. G. (2020) The evolution of business process management and the implementation of business process improvement initiatives. Proceedings of Voronezh State University. Series: Economics and Management. 3, 122-132. (In Russ., abstract in Eng.). DOI: 10.17308/econ.2020.3/3111

\begin{abstract}
Subject. The article outlines the evolution of business process management - from designing single business processes to a fledged management discipline and a nowadays understanding of business process management as an integrated set of organizational capabilities. The emphasis is on the analysis of the best practices for implementing projects of business process improvement.

Purpose is to analyze modern theoretical and applied aspects of business process management, the reasons for the failure and success of projects to improve business processes, define the optimal organization, structure and method of work for project teams to business process improvement.

Method. Literature review, analysis of applied research and case studies.

Result. Defines the conditions for organizing project work to improve business processes, the necessary qualifications of project team members, the general structure of project participants (project team, support team (pilot group), milestone committee, subproject teams) have been determined. The content of the stages of work to business processes improvement has been clarified.

Conclusion. The potential of business process management and initiatives to improve business processes can be realized if they are embedded in the most important components of an organization's management, such as strategy, structure, organizational culture, information and communication technologies, and personnel management. Equally important is the constant development of competencies, not only in the analysis and modeling of business processes, but also in project management and organizational change.
\end{abstract}

Key words: business process management, business process improvement, project team.

\section{Conflict of Interest}

The author declares that there are no obvious or potential conflicts of indifference related to the publication of this article.

Knowledge and Process Management. 22 (3), 180-190. DOI: $10.1002 / \mathrm{kpm} .1473$

2. Bakotich, D., \& Krnich, A. (2017) Exploring the relationship between business process improvement and employees' behavior. Journal of Organizational Change Management. 30 (4), 1044-1062.DOI: 10.1108/ jocm-06-2016-0116

3. Clark, K. (1998) Dynamic Manufacturing Creating the Learning Organization. New York, Free Press.

4. Crawford, L. (2006) Developing organizational project management capability: theory and practice. Project Management Journal. 37 (3), 74-97. DOI: 10.1177/875697280603700308

5. Davenport, T. H. (1993) Process Innovation: Reengineering Work through Information Technology. Boston, Harvard Business School Press.

\section{References}

1. Aarnio, T. (2015) The Strengthened Business Process Matrix - A Novel Approach for Guided Continuous Improvement at Service-Oriented SMEs.

6. Grover, V., Kettinger, W. J. \& Teng, J. T. C. (2000) Business process change in the 21st century. Business \& Economic Review, 46 (2), 14-18.

7. Grover, V. (1999) From business reengineering to business process change management: a longitudinal study of trends and practices. IEEE Transactions on Engineering Management, 46 (1), 36-46.

8. Harrington, H.J. (1991) Business Process Improvement - The Breakthrough Strategy for Total Quality. Productivity, and Competitiveness. New York, McGraw-Hill.

9. Hayes, R. H. (1984) Restoring Our Competitive Edge: Competing Through Manufacturing. New York, John Wiley.

10. Karimi, J., Somers, T. \& Bhattacherjee, A. (2007) The impact of ERP implementation on business pro- 
cess outcomes: a factor-based study. Journal of Management Information Systems, 24 (1), 101-134.

11. Jurisch, M., Palka, W., Wolf, P. \& Krcmar, H. (2014) Which capabilities matter for successful business process change? Business Process Management Journal, 20 (1), 47-67. DOI: 10.1108/bpmj-11-2012-0125

12. Leyer, M., Stumpf-Wollersheim, J. \& Kronsbein, D. (2017) Stains on the bright side of process-oriented organizational design: an empirical investigation of advantages and disadvantages. Schmalenbach Business Review, 17 (1), 29-47. DOI: 10.1007/s41464016-0020-9

13. Rosemann, M. (2010) The Service Portfolio of a BPM Center of Excellence. Handbook on Business Process Management. 2. 267-284. DOI: 10.1007/9783-642-01982-1_13

14. Rosemann, M., \& vom Brocke, J. (2010) The Six Core Elements of Business Process Management. Handbook on Business Process Management. 1. 107-122. DOI: $10.1007 / 978-3-642-00416-25$

15. Povey, B. (1998) The Development of a Best Practice Business Process Improvement Methodology.
Benchmarking for Quality Management and Technology. 5 (1). 27-44. DOI: 10.1108/14635779810206795

16. Sarker, S., \& Sidorova, A. (2006) Understanding business process change failure: an actor-network perspective. Journal of Management Information Systems, 23 (1), 51-86. DOI: 10.2753/mis0742-1222230102

17. van der Aalst, W. \& van Hee, K. (2002) Workflow Management: Models, Methods, and Systems. Cambridge, MIT Press.

18. vom Brocke, J., \& Rosemann, M. (2014) Business Process Management. Wiley Encyclopedia of Management, 7. 11-37. DOI: 10.1007/978-3-64245100-3

19. vom Brocke, J. \& Sinnl, T. (2011) Culture in business process management: a literature review. Business Process Management Journal. 17(2). 357-377. DOI: $10.1108 / 14637151111122383$

20. Zaini Z. \& Saad A. (2019) Business Process Reengineering as the Current Best Methodology for Improving the Business Process. Journal of ICT in Education. 6. 66-85.
Ruslan G. Pozhidaev, Candidate of Economic Science, Associate Professor, Associate Professor of the Labor Economy and General Management Department of the Voronezh State University, Voronezh, Russian Federation

E-mail: ruslan pozhidaev@mail.ru

ORCID: 0000-0002-5297-673X
Received 10.07.2020

Accepted 10.09.2020 